EPJ Web of Conferences 28, 06001 (2012)

DOI: $10.1051 /$ epjconf/20122806001

(c) Owned by the authors, published by EDP Sciences, 2012

\title{
Diboson Physics at the Tevatron
}

\author{
Aidan Robson ${ }^{1, a}$ for the CDF and D0 Collaborations \\ ${ }^{1}$ SUPA, School of Physics and Astronomy, University of Glasgow, G12 8QQ, Scotland
}

\begin{abstract}
Tevatron diboson measurements are reviewed, and new or recent results reported for $\mathrm{W} \gamma, \mathrm{Z} \gamma$ and $\mathrm{ZZ}$ production in the leptonic decay modes, and for $\mathrm{WW} / \mathrm{WZ}$ production in the lepton plus jets decay mode. The most stringent limits on anomalous triple gauge couplings are reported for each final state.
\end{abstract}

\section{Introduction}

Following the ending of the Tevatron collider program, we can review the significant progress that has been made in the diboson sector over the ten years of Run 2. At the start of Run 2, WW was the only massive diboson state to have been measured, with only a handful of events. In the intervening years, the $\mathrm{WZ}$ and $\mathrm{ZZ}$ processes have been observed (in 2007 and 2008 respectively), and the new availability of theoretical tools such as MCFM [1] and Mc@NLO [2] has allowed the standard model to be tested in the diboson sector. Measuring diboson production addresses the basic physics interest of observing fundamental electroweak processes. Measuring increasingly small cross-sections is a stepping-stone to new physics; and as diboson production is a major background to Higgs searches, it is important to understand it. Furthermore, measuring diboson production allows access to triple gauge couplings, which could provide indications of new physics.

\section{$2 \mathrm{~W} / \mathrm{Z}+$ photon}

\section{$2.1 \mathrm{~W} \gamma$}

D0 has a new result in $\mathrm{W} \gamma$ production from September this year, using $4.2 \mathrm{fb}^{-1}$ of integrated luminosity. Events are selected with an electron or muon, a photon, and missing transverse energy $\left(E_{T}\right)$. This analysis uses a neural network for photon identification to improve sensitivity to $\mathrm{WW} \gamma$ coupling. Backgrounds are at the $20-25 \%$ level, overwhelmingly $\mathrm{W}+$ jets, and are estimated from data. An important property of the standard model prediction at leading order is that interference between the $s$ - and $t$-channel amplitudes produces a zero in the total $\mathrm{W} \gamma$ yield at a specific angle $\theta^{*}$ between the $\mathrm{W}$ boson and the incoming quark in the $\mathrm{W} \gamma$ rest frame. Although it is difficult to measure the angle directly, this so-called 'radiation amplitude zero' is also visible in the charge-signed photon-lepton rapidity difference as a dip at around -1/3. Figure 1 shows the dip,

a e-mail: aidan.robson@glasgow.ac.uk compared with the signal prediction from the Baur-Berger dedicated event generator [3] interfaced to PYTHIA [4] for showering. The measured cross-section for the kinematic region $E_{T}(\gamma)>15 \mathrm{GeV}$ and $\Delta R(\ell \gamma)>0.7$ is

$\sigma(p \bar{p} \rightarrow \mathrm{W} \gamma+\mathrm{Z} \rightarrow \ell \gamma+\mathrm{X})=(7.6 \pm 0.4$ (stat) $\pm 0.6($ sys $)) \mathrm{pb}$ in good agreement with the standard model prediction 7.6 \pm $0.2 \mathrm{pb}$. If there were anomalous triple gauge couplings, the photon $E_{T}$ spectrum would be modified and more high- $E_{T}$ photons observed. The photon $E_{T}$ spectrum may therefore be used to derive limits on anomalous WW $\gamma$ couplings. A binned likelihood fit to data is used, and the 1-d limits $95 \% \mathrm{CL}$ limits obtained are $-0.4<\Delta \kappa_{\gamma}<0.4$ and $-0.08<\lambda_{y}<0.07$ for a new physics scale $\Lambda=2 \mathrm{TeV}$.

\section{$2.2 \mathrm{Z} \gamma$}

D0 has a new result for this conference in $\mathrm{Z} \gamma$, using $6.2 \mathrm{fb}^{-1}$ of integrated luminosity. Again, a neural network technique that uses five variables from tracking, calorimetry, and the preshower detectors provides robust differentiation between photons and jets. Background is at the 5-10\% level and is dominated by $\mathrm{Z}+$ jets. Around 1000 events are observed in each of the final states $\mathrm{Z} \rightarrow \mathrm{e}^{+} \mathrm{e}^{-}+\gamma$ and $\mathrm{Z} \rightarrow \mu^{+} \mu^{-}+\gamma$. The $\mathrm{Z} \gamma$ system has the property that initial state photon radiation (ISR) may be selected preferentially over final state photon radiation by requiring the three-body invariant mass $M(\ell \ell \gamma)$ to be above the $\mathrm{Z}$ boson mass. With $M(\ell \ell \gamma)>$ $110 \mathrm{GeV} / c^{2}$, around 300 events are observed in each of the final states. The differential cross-section $d \sigma / d p_{T}(\gamma)$ is measured, using matrix inversion to unfold the experimental distribution, and is shown in Figure 2 both for all $M(\ell \ell \gamma)$, and for the ISR-dominated sample $M(\ell \ell \gamma)>$ $110 \mathrm{GeV} / c^{2}$. Prior to this analysis, these differential distributions had not been shown. The data are compared with the NLO prediction from MCFM, and are seen to be consistent. Total cross-sections are also quoted: for the kinematic region $|\eta(\gamma)|<1, E_{T}(\gamma)>10 \mathrm{GeV}, \Delta R(\ell \gamma)>0.7$ and $M(\ell \ell \gamma)>60 \mathrm{GeV} / c^{2}$ the result is $\sigma(p \bar{p} \rightarrow \mathrm{Z} \gamma \rightarrow \ell \ell \gamma)=$ $(1.09 \pm 0.04$ (stat) \pm 0.07 (sys) $) \mathrm{pb}$, to be compared with the standard model prediction $1.10 \pm 0.03 \mathrm{pb}$; and for $M(\ell \ell \gamma)>$ $110 \mathrm{GeV} / c^{2}$ the result is $\sigma(p \bar{p} \rightarrow \mathrm{Z} \gamma \rightarrow \ell \ell \gamma)=(0.29 \pm$ 

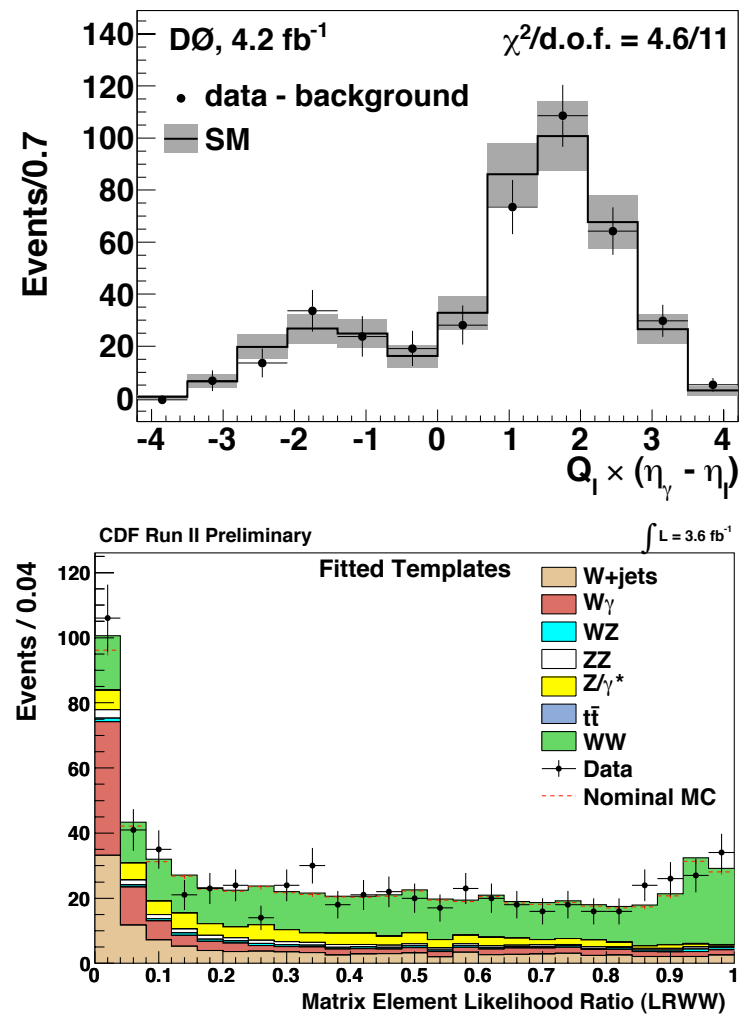

Fig. 1. (upper) The charge-signed photon-lepton rapidity difference for $\mathrm{W} \gamma$ candidates from D0, showing the radiation amplitude zero as a dip at around $-1 / 3$; and (lower) the matrix element likelihood discriminant for WW candidates from CDF.

0.02 (stat) \pm 0.01 (sys)) pb, to be compared with the standard model prediction $0.29 \pm 0.01 \mathrm{pb}$

The most stringent anomalous coupling limits in $\mathrm{Z} \gamma$ are from CDF, in another 2011 result [5]. Here, $\mathrm{Z} \rightarrow \ell^{+} \ell^{-}+\gamma$ events are selected with $M(\ell \ell \gamma)>100 \mathrm{GeV} / c^{2}$, and $\mathrm{Z} \rightarrow \nu v$ is also included through events having $E_{T}>50 \mathrm{GeV}$. For the $\mathrm{Z} \rightarrow v v+\gamma$ selection, events with tracks having $p_{T}>$ $10 \mathrm{GeV}$ or jets having $E_{T}>15 \mathrm{GeV}$ are rejected, and calorimeter timing information is used to reject cosmic-ray tracks. The photon $E_{T}$ spectrum shows no evidence for anomalous couplings and is used to set limits; at 95\% CL they are $-0.020<h_{3}^{Z}<0.021,-0.0009<h_{4}^{Z}<0.0009$, $-0.022<h_{3}^{\gamma}<0.020$, and $-0.0008<h_{4}^{\gamma}<0.0008$, for $\Lambda=1.5 \mathrm{TeV}$.

D0 has also used the $\mathrm{Z} \gamma$ signature to look for resonances and has set limits on generic scalar or vector resonances, such as might occur in technicolour models, at the level of $1 \mathrm{pb}[6]$.

\section{Massive Dibosons}

\subsection{WW}

The WW final state is intimately connected with Higgs searches, and CDF's WW measurement was done in paral-
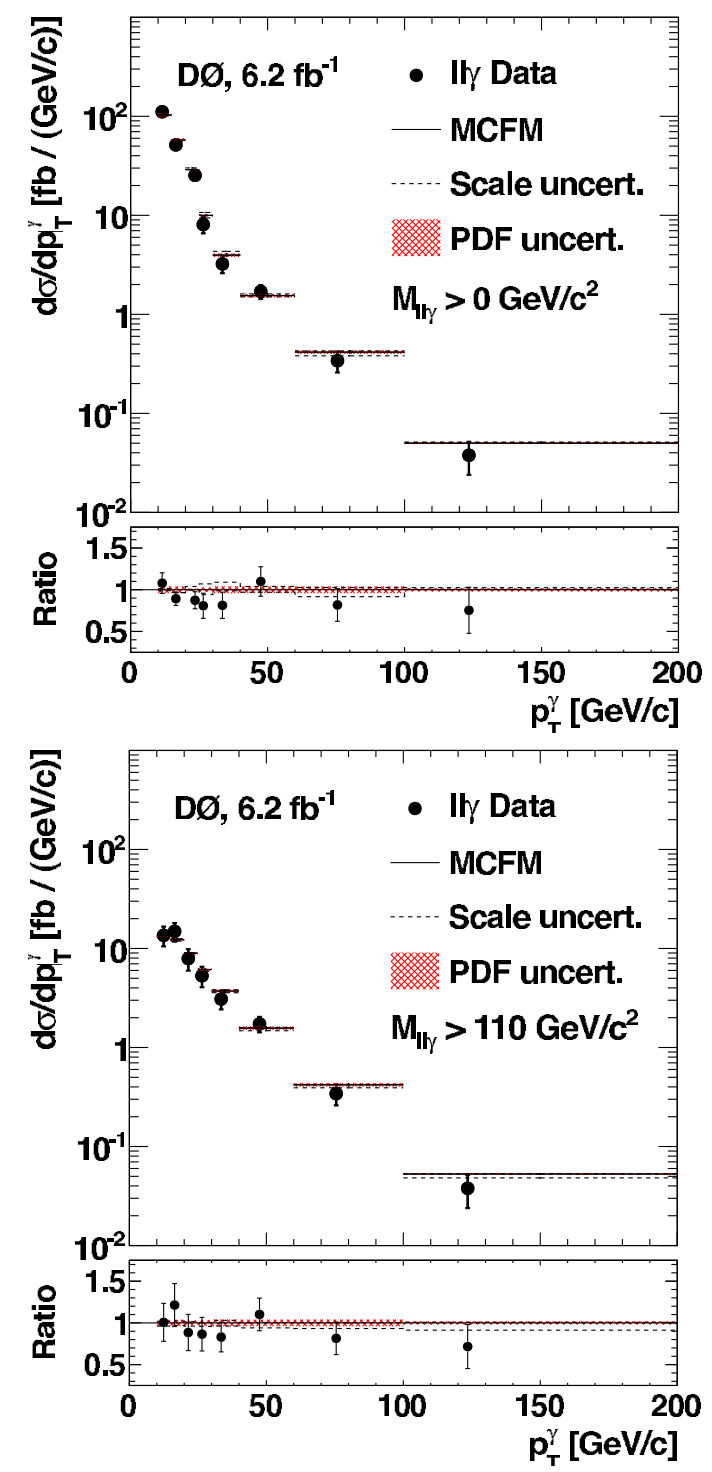

Fig. 2. The differential cross-section $d \sigma / d p_{T}(\gamma)$ for $\mathrm{Z} \gamma$ events from D0, for (upper) all values of $M(\ell \ell \gamma)$, and (lower) the ISRenhanced dataset $M(\ell \ell \gamma)>110 \mathrm{GeV} / c^{2}$.

lel with the search for $\mathrm{H} \rightarrow \mathrm{WW}$, using the same tools [7]. Events having two oppositely-charged leptons and $E_{T}$ are selected. Around $12 \%$ of the acceptance comes from $\tau$ leptons decaying to electrons or muons. Control samples such as same-sign dileptons check background modelling. The analysis uses a matrix element probability approach, where transfer functions derived from simulation are applied to the measured four-vectors, which are inputs to matrix elements that allow the computation of probabilities that an event comes from signal or one of several background processes. These probabilities are put together in a likelihood ratio, and the cross-section is extracted from a fit as shown in Figure 1. With $3.6 \mathrm{fb}^{-1}$ of integrated luminosity, CDF measures $\sigma(p \bar{p} \rightarrow \mathrm{WW})=\left(12.1 \pm 0.9(\mathrm{stat})_{-1.4}^{+1.6}(\mathrm{sys})\right) \mathrm{pb}$. A small excess in the high tail of the lepton $p_{T}$ spectrum makes anomalous triple gauge coupling limits less stringent than expected. The probability that the observed dis- 
tribution is drawn from the standard model is not too small (7\%), so the small excess is ascribed to a statistical fluctuation. The best limits come from D0's $1 \mathrm{fb}^{-1}$ analysis [8], and at $95 \% \mathrm{CL}$ are $-0.54<\Delta \kappa_{\gamma}<0.83,-0.14<\lambda_{\gamma}=$ $\lambda_{Z}<0.18$, and $-0.14<\Delta g_{1}^{Z}<0.30$ for a new physics scale $\Lambda=2 \mathrm{TeV}$.

\section{$3.2 \mathrm{WZ}$}

The WZ final state is little-studied as it is charged, and therefore produced only at hadron colliders. CDF's recent analysis in the $\ell \ell \ell v$ final state using $6 \mathrm{fb}^{-1}$ of integrated luminosity incorporates improvements in lepton selection and shows very good resolution, as demonstrated by the $\mathrm{W}$ boson transverse mass in Figure 3. The measured crosssection is normalised to the measured $\mathrm{Z}$ boson cross-section to remove some systematic uncertainties, in particular the luminosity uncertainty. This is then exchanged for a smaller theoretical uncertainty when multiplying by a calculation of the $\mathrm{Z}$ boson production cross-section in order to recover the WZ cross-section: $\sigma(p \bar{p} \rightarrow \mathrm{WZ}) / \sigma(p \bar{p} \rightarrow \mathrm{Z})=$ $(5.5 \pm 0.9) \times 10^{-4}$ and $\sigma(p \bar{p} \rightarrow \mathrm{WZ})=(4.1 \pm 0.7) \mathrm{pb}$. Triple gauge couplings were not studied in this analysis and the best anomalous coupling limits are set by D0's $4.1 \mathrm{fb}^{-1}$ analysis, using the $\mathrm{Z}$ boson $p_{T}$ distribution shown in Figure 3: $-0.400<\Delta \kappa_{Z}<0.675,-0.077<\lambda_{Z}<0.093$, and $-0.056<\Delta g_{1}^{Z}<0.154$ for a new physics scale $\Lambda=2 \mathrm{TeV}$ [9].

\section{$3.3 \mathrm{ZZ}$}

$\mathrm{CDF}$ has a new measurement of the $\mathrm{ZZ}$ production crosssection in the four-lepton final state using $6 \mathrm{fb}^{-1}$ of integrated luminosity [11]: $\sigma(p \bar{p} \rightarrow \mathrm{ZZ})=\left(2.3_{-0.8}^{+0.9}(\right.$ stat $) \pm$ 0.2 (sys) $) \mathrm{pb}$, to be compared with the NLO standard model prediction $1.4 \pm 0.1 \mathrm{pb}$. A clustering of events at high mass, shown in Figure 4, caused excitement. However, analysis of the other $\mathrm{ZZ}$ final states $\mathrm{ZZ} \rightarrow \ell^{+} \ell^{-} v v$ and $\mathrm{ZZ} \rightarrow \ell^{+} \ell^{-} \mathrm{jj}$ showed them to be more sensitive to a resonance of mass around $327 \mathrm{GeV} / c^{2}$ decaying to $\mathrm{ZZ}$, and the data in those channels are in agreement with standard model predictions. The four-lepton events therefore appear to arise from standard model sources.

$\mathrm{D} 0$ also has a recent measurement of $\mathrm{ZZ} \rightarrow \ell^{+} \ell^{-} \ell^{+} \ell^{-}$, with increased muon acceptance compared to previous results [12]. The measured cross-section is $\sigma(p \bar{p} \rightarrow \mathrm{ZZ})=$ $\left(1.26_{-0.37}^{+0.47}\right.$ (stat) \pm 0.14 (sys) $) \mathrm{pb}$. The distribution in angle between the planes of the lepton pairs, computed in the $\mathrm{ZZ}$ rest-frame, is sensitive to the production mechanism of the $\mathrm{Z}$ pair: for example, a $\mathrm{Z}$ pair arising from the decay of a Higgs boson would result in a different angular distribution. This distribution is tested for the first time and is shown in Figure 4; it is seen to be consistent with the standard model expectation.

The only anomalous triple coupling limits from $\mathrm{ZZ}$ are from an earlier D0 result [13]: $-0.28<f_{40}^{Z}<0.28,-0.26<$ $f_{40}^{\gamma}<0.26,-0.31<f_{50}^{Z}<0.29$, and $-0.30<f_{50}^{\gamma}<0.28$ at $95 \% \mathrm{CL}$ for $\Lambda=1.2 \mathrm{TeV}$.
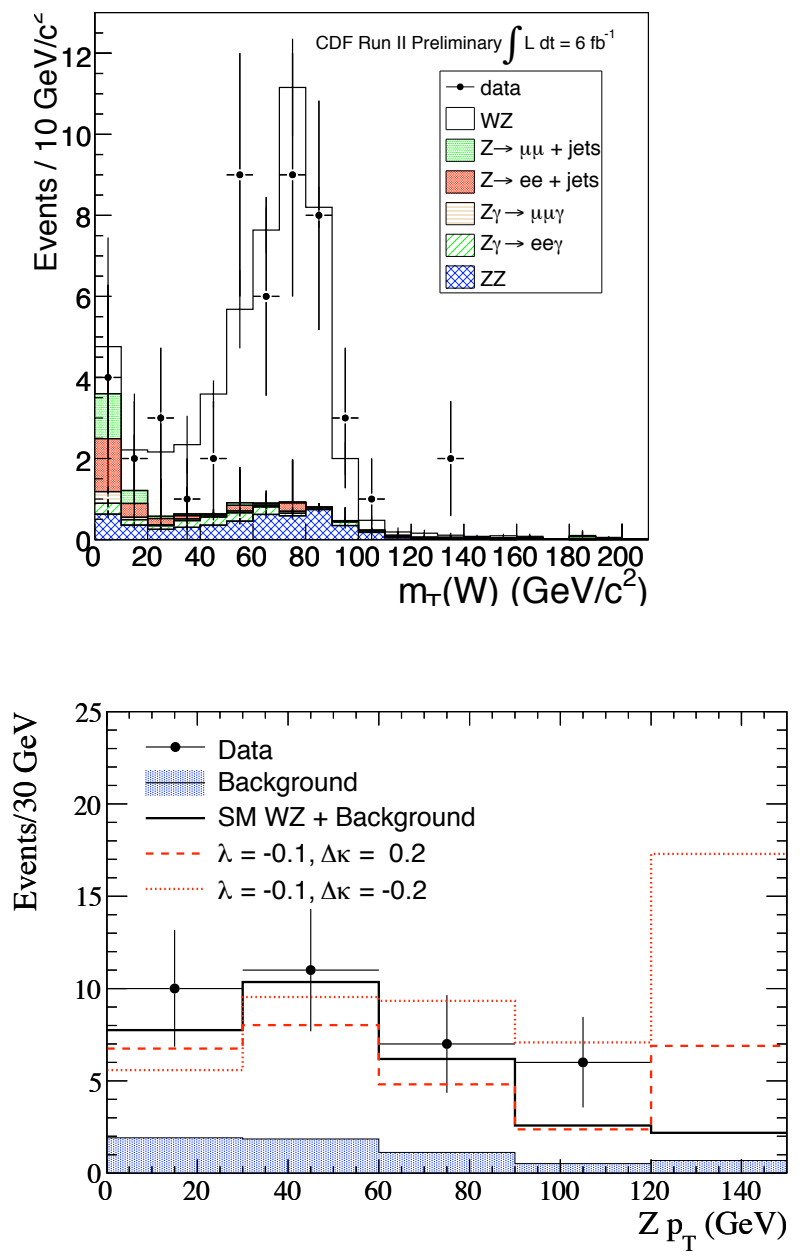

Fig. 3. (upper) The $\mathrm{W}$ boson transverse mass $m_{T}$ in WZ candidates recorded by CDF; and (lower) the $\mathrm{Z}$ boson $p_{T}$ in $\mathrm{WZ}$ candidates recorded by D0.

Finally, CDF has measured $\mathrm{ZZ} \rightarrow \ell^{+} \ell^{-} v v$ in $5.9 \mathrm{fb}^{-1}$ of integrated luminosity using techniques from the Higgs search, and in that channel found $\sigma(p \bar{p} \rightarrow \mathrm{ZZ})=\left(1.45_{-0.42}^{+0.45}(\mathrm{stat})_{-0.30}^{+0.41}(\mathrm{sys})\right) \mathrm{pb}$.

\section{Diboson final states with jets}

Given their similarity to key Higgs boson signatures, there have been ongoing efforts to observe diboson production in final states with jets.

Two CDF analyses observed WW and WZ production in the $\ell v j j$ final state in 2010 . This final state is very similar to that expected from $\mathrm{WH}$ associated production. $\mathrm{W}+\mathrm{jets}$ is the overwhelming background. In the first analysis, the background contribution from QCD was fitted from data using the $E_{T}$ distribution, where for the analysis selection, QCD enters at low values, and electroweak processes have high values. The signal was extracted from a $\chi^{2}$ fit to the dijet mass distribution as shown in Figure 5, giving an extracted cross-section $\sigma(\mathrm{WW}+\mathrm{WZ})=(18.1 \pm 3.3($ stat $) \pm$ 2.5 (sys)) pb with $5.2 \sigma$ significance [14]. The second analysis used a matrix element technique, for which the final 
EPJ Web of Conferences
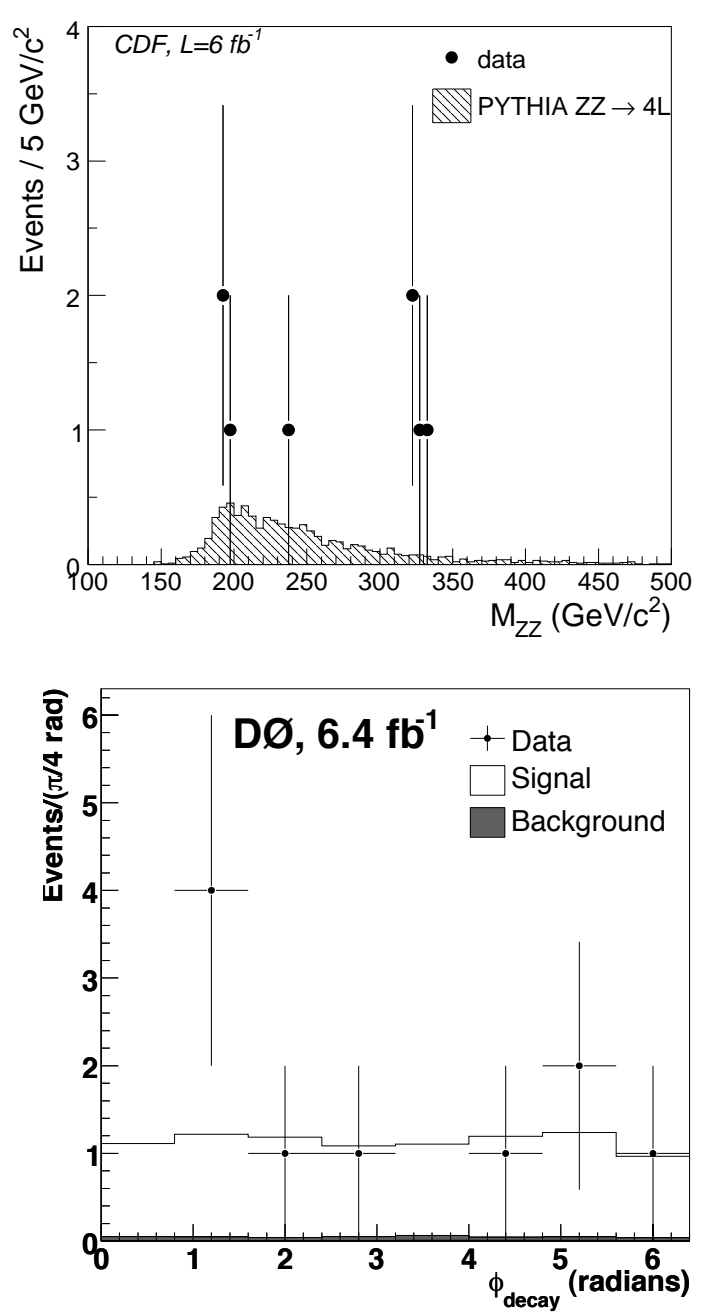

Fig. 4. (upper) The reconstructed four-lepton mass in CDF $\mathrm{ZZ} \rightarrow \ell^{+} \ell^{-} \ell^{+} \ell^{-}$candidates, and (lower) angular separation of the lepton pair planes, measured in the $\mathrm{ZZ}$ rest frame, for $\mathrm{D} 0$ $\mathrm{ZZ} \rightarrow \ell^{+} \ell^{-} \ell^{+} \ell^{-}$candidates.

event probability discriminant is shown in Figure 5. Here, the extracted cross-section was $\sigma(\mathrm{WW}+\mathrm{WZ})=16.5_{-3.0}^{+3.3} \mathrm{pb}$, with $5.4 \sigma$ significance [14].

New for this conference is D0's latest update in the $\ell v j j$ final state, using $4.3 \mathrm{fb}^{-1}$ of integrated luminosity, which makes significant advances. A random forest multivariate discriminant is used to separate signal from background, and since $\mathrm{Z}$ bosons can decay to $\mathrm{b}$-quark pairs but $\mathrm{W}$ bosons cannot, $b$-tagging is employed both to improve the significance of the observation, and to separate the WW and WZ components. Both the random forest discriminant output, and the dijet invariant mass for the no $b$-tag data sample, are shown in Figure 6. A cross-section $\sigma(\mathrm{WW}+\mathrm{WZ})=$ $19.6_{-3.0}^{+3.1} \mathrm{pb}$ is measured, with $8 \sigma$ significance, and contours of the separated WW and WZ cross-sections are given in Figure 6.

Further results of diboson analyses with decays to bquark pairs are given elsewhere in these proceedings [17].

An earlier version of this D0 analysis using $1 \mathrm{fb}^{-1}$ of integrated luminosity set limits on anomalous triple cou-
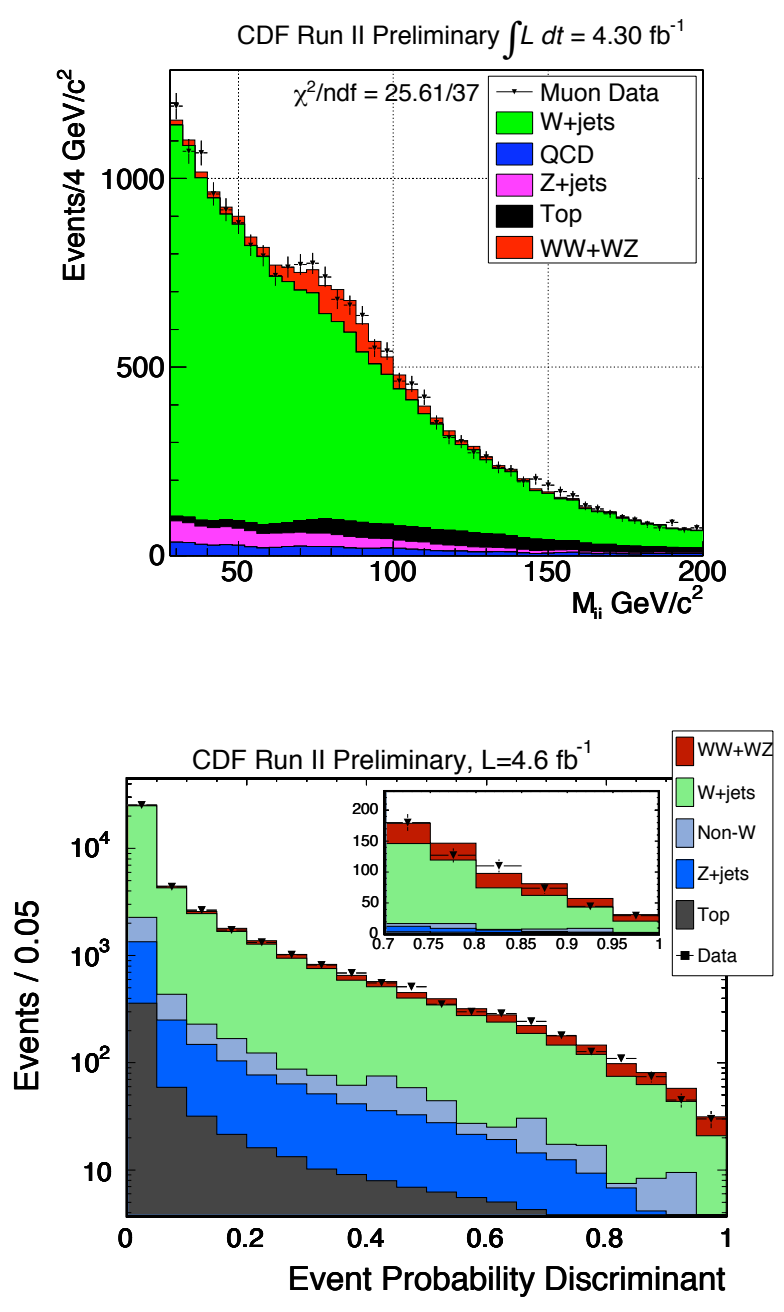

Fig. 5. (upper) The dijet invariant mass spectrum in the WW/WZ muon+jets channel from CDF, and (lower) the event probability discriminant in the matrix-element probability approach.

plings: $-0.44<\Delta \kappa_{\gamma}<0.675,-0.10<\lambda_{Z}=\lambda_{\gamma}<0.11$, and $-0.12<\Delta g_{1}^{Z}<0.20$ at $95 \% \mathrm{CL}$ for $\Lambda=2 \mathrm{TeV}$ [16].

Finally, an early analysis from $\mathrm{CDF}$ in $\mathrm{ZZ} \rightarrow \ell^{+} \ell^{-}$jj using $1.9 \mathrm{fb}^{-1}$ of integrated luminosity set anomalous coupling limits: at $95 \% \mathrm{CL},-0.12<f_{4}^{Z}<0.12,-0.10<$ $f_{4}^{\gamma}<0.10,-0.13<f_{5}^{Z}<0.12$, and $-0.11<f_{5}^{\gamma}<0.11$ for $\Lambda=1.2 \mathrm{TeV}$.

\section{Outlook}

A rich programme of Tevatron diboson physics has made huge advances over the ten years of Run 2, testing the standard model, probing for new physics, and underpinning electroweak symmetry-breaking searches. D0 combined anomalous coupling limits with $1 \mathrm{fb}^{-1}$ of integrated luminosity, resulting in more stringent limits. Some of those have now been superseded, and there is work on a new combination. Both experiments have a final dataset of around $10 \mathrm{fb}^{-1}$, so as well as being combined, these analyses should be updated once more for legacy measurements. 

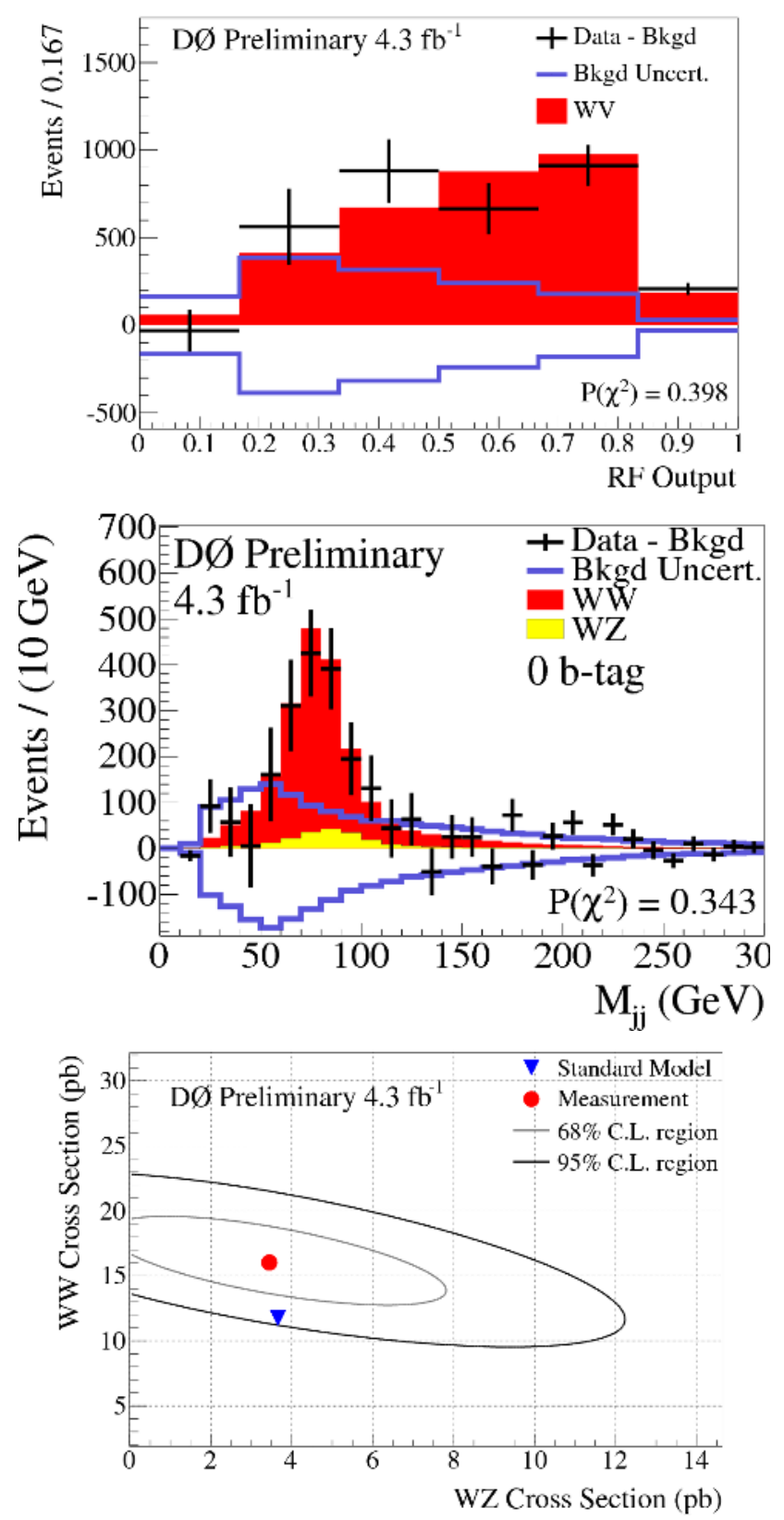

Fig. 6. Results from D0's WW/WZ analysis in the $\ell v j j$ final state: (upper) random forest multivariate discriminant output; (centre) background-subtracted dijet mass; (lower) contours of WW and $\mathrm{WZ}$ production cross-section.

\section{References}

1. J. M. Campbell and R. K. Ellis, Phys. Rev. D 60, 113006 (1999).

2. S. Frixione and B. R. Webber, J. High Energy Phys. 0206, 029 (2002).

3. U. Baur, T. Han, J. Ohnemus, Phys. Rev. D 48, 5140 (1993).

4. T. Sjöstrand et al., Comput. Phys. Commun. 135, 238 (2001).

5. T. Aaltonen et al. (CDF Collaboration), Phys. Rev. Lett. 107, 051802 (2011).
6. V. M. Abazov et al. (D0 Collaboration), Phys. Lett. B 671349 (2009).

7. T. Aaltonen et al. (CDF Collaboration), Phys. Rev. Lett. 104, 201801 (2010).

8. V. M. Abazov et al. (D0 Collaboration), Phys. Rev. Lett. 103, 191801 (2009).

9. V. M. Abazov et al. (D0 Collaboration), Phys. Lett. B 69567 (2011).

10. V. M. Abazov et al. (D0 Collaboration), Phys. Rev. Lett. 107, 011801 (2011).

11. http://arxiv.org/abs/1111.3432

12. V. M. Abazov et al. (D0 Collaboration), Phys. Rev. D 84, 011103 (2011).

13. V. M. Abazov et al. (D0 Collaboration), Phys. Rev. Lett. 100, 131801 (2008).

14. T. Aaltonen et al. (CDF Collaboration), Phys. Rev. Lett. 104, 101801 (2010).

15. T. Aaltonen et al. (CDF Collaboration), Phys. Rev. D 82, 112001 (2010).

16. V. M. Abazov et al. (D0 Collaboration), Phys. Rev. D 80, 053012 (2009).

17. J.-F. Grivaz, Dibosons with $\mathrm{Z} \rightarrow \mathrm{bb}$ at the Tevatron; these proceedings. 